
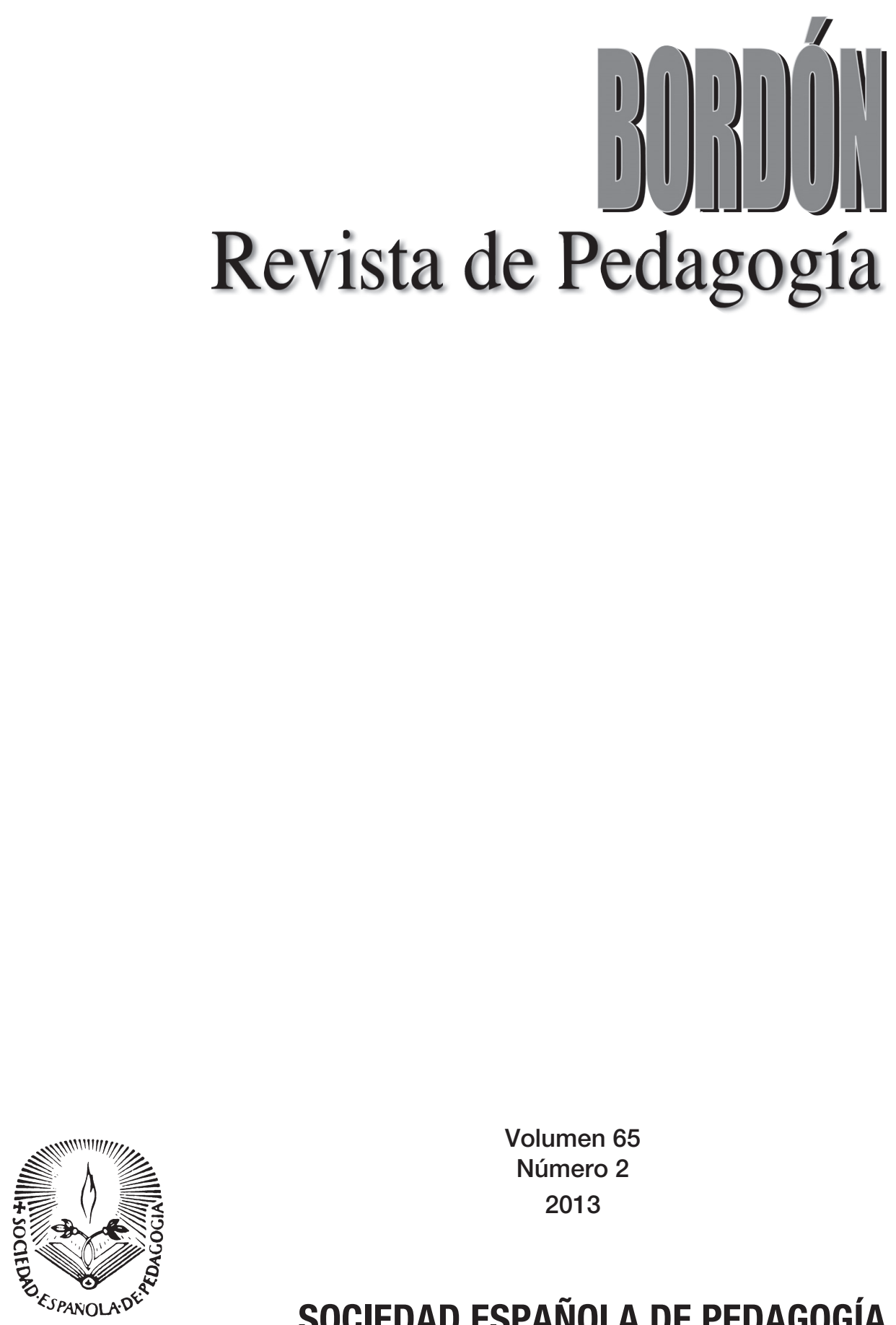

Volumen 65

Número 2

2013

SOCIEDAD ESPAÑOLA DE PEDAGOGÍA 



\section{DE LA TEORÍA A LA PRÁCTICA: EL CURRÍCULUm DE EDUCACIÓn FÍSICA EN LAS ESCUELAS DE EDUCACIÓn PRIMTARIA UENEZOLAMAS}

\section{From Theory to Practice: the Physical Education Curriculum in Primary Schools in Uenezuela}

ISABEL CANTÓN MAYO

Universidad de León

ARGENIRA CARLOTA RAMOS DE BALAZS

Universidad Pedagógica Libertador de Venezuela

INTRODUCCIÓN. El objetivo de esta investigación es conocer la similitud o la discrepancia entre el currículum prescrito y el currículum real de aula en la materia de Educación Física en las Escuelas Primarias de Venezuela. Para ello se realiza una revisión del tema desde la perspectiva curricular general englobando las tres corrientes habituales: técnica, interpretativa o hermeneútica y crítica. Luego se revisan y describen las propuestas y diseños curriculares normativos en el estado de Venezuela para Educación Primaria, que se enmarcan en las tres dimensiones citadas, con predominio de la perspectiva técnica. MÉTODO. El método de investigación fue de evaluación de programas con el modelo CIPP de Stuflebeam y se utilizaron cuestionarios que se aplicaron a una muestra representativa de docentes complementados con instrumentos cualitativos como la observación y la entrevista. Se evaluaron aspectos como la aptitud física, el ritmo corporal, la vida al aire libre y la expresión y comunicación corporal en tres niveles: la planificación de la enseñanza, la relación entre la práctica de aula y el diseño curricular prescrito y la propuesta de acciones de mejora. RESULTADOS. Los resultados muestran que la distancia entre lo prescrito y lo realizado es amplia y diferente para las distintas categorías analizadas, dentro del currículum de Educación Física. DISCUSIÓN. El artículo es relevante por mostrar la necesidad de acercar la realidad de las aulas a la normativa. La representatividad de la muestra sería mayor si se replicase la investigación en otros estados de Venezuela.

Palabras clave: Currículum prescrito, Currículum realizado, Evaluación de programas, Educación Primaria, Juegos motrices, Aptitud física, Ritmo corporal, Vida al aire libre. 


\section{Principales tendencias en el estudio del currículum}

\section{Pertinencia de la Teoría Curricular}

El currículum, como realidad pedagógica que evoluciona, debe ser revisado bajo el condicionamiento directo del desarrollo de la vida escolar y requiere una fundamentación científica. Tal acotación, sin embargo, ha sido en oportunidades motivo de polémica. Algunos de sus defensores la han llevado al plano de la exigencia. Tal es el caso de Gervilla (2006: 11) quien se expresa, al respecto, de la siguiente manera:

«No obstante, si para orientar la práctica educativa debemos partir de un análisis de las condiciones previas y específicas que presenta la realidad sobre la que se va a actuar, está claro que debemos separamos de lo inmediato y evidente mediante la teorización sí queremos analizar y comprender las necesidades y condicionamientos de dicha realidad a fin de orientar, lo mejor posible, el proceso enseñanza-aprendizaje.»

El estudio de currículum viene condicionado por los diferentes supuestos pedagógicos e ideológicos con los que se aborda, varios son los autores que han intentado clasificar las tendencias en el estudio de este ámbito, y aunque nosotros nos acogemos a la perspectiva curricular (Román y Diez, 2003), recogemos sintetizadas las aportaciones de diversos autores sobre este tema englobando las principales corrientes: técnica, hermeneútica o simbólica y sociocrítica: Gimeno (1995), Gagné (1990), Taba (1991), Saylor (1970), Wheeler (1993), Pinar (1999), Zabalza (1991), Castro, Correa y Lira (2004), Rodríguez (1997), Habermas (1998), Giroux (1990), Apple (1986), Popkewitz, (1994), McTaggart y Singh (1986), Kemmis (1996), Espinoza (2001), Bernstein (2000), Cantón y Pino (2011).
Hay diferentes posiciones sobre el currículum: desde plantearlo como un proceso técnico, donde los expertos diseñan la tarea que los profesores llevarán a la práctica, buscándose con ello una máxima eficiencia, hasta proponerlo como el estudio que realizan los mismos profesores sobre la realidad práctica, con el fin de intervenir en el contexto social en la que esta se desarrolla. Entre una postura y la otra se han ido estableciendo posiciones de diferentes autores.

En la planificación didáctica se pretende tener presente la selección y organización de los contenidos y considerar los conocimientos de entrada de los estudiantes, para programar el aprendizaje. El currículum se visualiza entonces como la normativa legal, flexible, que contiene principios y orientaciones sobre los contenidos y los procesos para llevar a cabo la práctica escolar, así como también para propiciar el desarrollo personal y la inserción del individuo en la sociedad por medio de la enseñanza y del aprendizaje.

\section{Discrepancias sobre el currículum básico, oficial y prescriptivo}

Parece evidente que el fenómeno de escolarización generalizada de la población, impulsada generalmente por intereses estatales de normalizar una enseñanza de una forma acorde a los objetivos económicos y sociales, genera la necesidad de establecer un currículum oficial básico y prescriptivo (Tjeerdsma Blankenship, 2012). Surgen así, en los diversos países, propuestas de currículum iguales para toda la población y a la vez de cumplimiento obligatorio, que por lo general son objeto de fuertes críticas.

Con relación a este tema, recogemos los argumentos recogidos por Coll (1989: 8), a favor y en contra de la existencia de bases curriculares comunes: 
De la teoría a la práctica: el currículum de Educación Física en las Escuelas de Educación Primaria Venezolanas

\section{CuAdro 1. Argumentos a favor y en contra de la existencia de bases curriculares comunes}

\section{Argumentos a favor}

- Asegurar a todos los estudiantes el acceso, a lo largo de su escolaridad, a un abanico de contenidos amplio y equilibrado, de manera que no abandonen demasiado pronto unos estudios que pueden serles útiles más tarde y pueden contribuir a desarrollar su capacidad para adaptarse y responder de manera flexible a un mundo en constante cambio.

- Establecer unos objetivos de la educación obligatoria accesibles a todos los estudiantes, cualesquiera que sean sus capacidades.

- Asegurar que todos los estudiantes, con independencia del sexo, origen étnico, lugar de residencia y otras características individuales y sociales puedan cursar un currículum básicamente similar, relevante, vinculado a la experiencia propia valioso para la vida adulta.

- Disponer de un instrumento que permita valorar el progreso realizado por los estudiantes en los sucesivos niveles de la escolaridad obligatoria con el fin de exigir más a los que puedan avanzar más y proporcionar mayor ayuda pedagógica a los que la necesiten.

- Asegurar la progresión, coherencia y la continuidad en el transcurso de la educación obligatoria.

- Asegurar que el currículum impartido en todas las instituciones escolares posea elementos comunes suficientes para permitir a los estudiantes cambiar de escuela sin sufrir desajustes innecesarios.
Argumentos en contra

- Se opone al derecho de los estudiantes a seleccionar las actividades y los contenidos de aprendizaje de acuerdo con sus motivaciones e intereses.

- En la medida en que determina lo que han de aprender todos los estudiantes sin distinción, constituye un obstáculo para dar una respuesta educativa que tenga en cuenta la diversidad de capacidades, intereses y motivaciones de los estudiantes.

- Traduce necesariamente las preferencias y los valores del grupo o grupos sociales dominantes, puesto que es imposible llegar a un acuerdo de toda la sociedad sobre lo que han de aprender los estudiantes en las escuelas.

- No respeta el pluralismo cultural, que es un rasgo distintivo de las sociedades democráticas modernas.

- Implica una centralización y una burocratización de las decisiones educativas, que se alejan así irremediablemente de las necesidades reales de los estudiantes y de la comunidad escolar en su conjunto.

- Anula la autonomía y la iniciativa profesional de los docentes, quedan convertidos en simples ejecutores de un plan previamente establecido.
Coll expone estos argumentos al referirse a la polémica establecida en el Reino Unido en relación a la implementación del National Curriculum. Es difícil imaginar un país sin directrices curriculares básicas y comunes para toda la población. Garantizar el acceso a una educación básica para todos es responsabilidad de cualquier país democrático. Legislar una selección y organización de objetivos y contenidos culturalmente equilibrados que deban llegar a la totalidad de la población constituye un medio para crear mecanismos para atenuar desigualdades entre sus miembros. Esta legislación sobre el currículum supone, igualmente, un intento de favorecer la igualdad de oportunidades entre las personas. Resaltamos el carácter de intención que impregna esta igualdad de oportunidades, pues es necesario entender, como indica Gimeno (1995: 133), que no podemos caer en la ingenuidad de creer que, al estar regulados legalmente unos mínimos curriculares, la potencialidad de garantizar una igualdad de oportunidades se cumplirá necesariamente. Dependerá, obviamente, de forma más directa, 
de los procedimientos y medios con los que se hace efectiva esa cultura común.

\section{Los diseños curriculares de la I y II Etapa de Educación Básica en EF}

Las directrices del Ministerio de Educación de Venezuela en el Currículo Básico Nacional de I y II Etapa de Educación Básica (1997) se mantienen vigentes en las Escuelas Bolivarianas e implican reflexionar sobre las concepciones, las metas y los propósitos de la educación venezolana. Están basados en documentos y diagnósticos realizados en el país, como son: el Informe de la Comisión Presidencial para el estudio del Proyecto Nacional (1986), el Diagnóstico del Banco Mundial (1992), la Reforma una Prioridad Nacional (1994) y el Plan de Acción del Ministerio de Educación (1995).

La Educación Física en Venezuela se hace pública el 9 de julio de 1980 en la Ley Orgánica de Educación en el artículo 12 en el que se declaran obligatorios la educación física y el deporte en todos los niveles y modalidades del sistema educativo, el Ejecutivo Nacional es el ente responsable de promover su difusión y práctica en todas las comunidades de la nación.

En ellos se contemplan varias teorías de aprendizaje: la integral sistémica, sustentada en el humanismo, la teoría genética de Jean Piaget (1979), la teoría sociocultural de Vigotski, la teoría del aprendizaje de Ausubel (1981), la teoría de procesamiento de la información, las teorías neurofisiológicas y la concepción constructivista. La teoría de Piaget se ve reflejada cuando se contempla la edad cronológica por niveles, así como también en el eje transversal del desarrollo del pensamiento. Los diseños en la I y II Etapa de Educación Básica contienen seis áreas académicas: Lengua y Literatura, Matemática, Educación Física, Educación Estética, Ciencias de la Naturaleza y Tecnología y Ciencias Sociales. En este trabajo nos centramos en el diseño de Educación Física.
La Educación Física plantea situaciones especialmente favorables a la adquisición de las competencias social y ciudadana. Las actividades físicas, propias de esta materia, son un medio eficaz para facilitar la integración y fomentar el respeto, a la vez que contribuyen al desarrollo de la cooperación, la igualdad y el trabajo en equipo. El cumplimiento de las normas y reglamentos que rigen las actividades deportivas colaboran en la aceptación de los códigos de conducta propios de una sociedad (Currículum Básico, 1997).

La Educación Física ayuda a la consecución de la competencia para aprender al ofrecer recursos para la planificación de determinadas actividades físicas a partir de un proceso de experimentación. Todo ello permite que el estudiante sea capaz de regular su propio aprendizaje y práctica de la actividad física en su tiempo libre, de forma organizada y estructurada.

El Ministerio de Educación (1996), cuando se refiere a la enseñanza de la Educación Física, la define como:

«Parte del proceso educativo que, a través de la actividad física planificada, promueve cambios persistentes de conducta a nivel afectivo, cognoscitivo y primordialmente psicomotor, para el logro de un individuo físicamente apto, desarrollado integralmente y plenamente integrado a la sociedad donde se desenvuelve» (p. 1).

Y además le establece los siguientes propósitos:

«1. Promover la adquisición y desarrollo de hábitos, habilidades, aptitudes de tipo psicomotor, que contribuyan al desarrollo pleno, armónico e integral de la personalidad del individuo y al mejoramiento de su salud física, mental y social. 2. Propiciar la adquisición de conocimientos y la formación de actitudes y valores referidos a la actividad física y la conservación del 
ambiente natural, que permitan al individuo el uso adecuado de su tiempo libre, en función del mejoramiento permanente de su calidad de vida y la de su comunidad» (p. 1).

Lo que se reitera, en un plano más operativo en el programa de la II Etapa de Educación Básica (1997: 2), que establece que en la puesta en práctica en el área de la Educación Física «se ocupa del mejoramiento total del ser humano, no solo relacionado con su capacidad de movimiento o lo que tenga relación con su cuerpo sino de sus capacidades como ser viviente y como ser cultural».

\section{Vinculación de los diseños curriculares con las prácticas docentes}

En los diseños curriculares se contemplan tres tipos de contenidos: conceptuales, Estos tipos de contenidos se relacionan con los aprendizajes fundamentales y con los tipos de capacidades: cognitivas-intelectuales, cognitivas-motrices, cognitivas-afectivas.

Básicamente están estructurados por bloques de contenido como elementos organizadores de las distintas áreas académicas. En el área de Educación Física, los bloques son: juegos motrices, aptitud física, ritmo corporal y vida al aire libre, y en la II Etapa de Educación Básica se incorpora el contenido de expresión y comunicación corporal.

Los juegos motrices son una actividad motriz de forma organizada de aprendizaje que tiene una evolución a lo largo de la etapa evolutiva del niño/a que va desde las formas espontáneas a las más especializadas por medio de reglas. Asimismo constituyen un medio para integrar e interrelacionar contenidos propios de la Educación Física con otras áreas. También fomenta la afirmación de la personalidad. En este bloque se abarca los siguientes contenidos: juegos de destrezas básicas, juegos tradicionales, juegos deportivos, capacidades físicas, flexibilidad, capacidad aeróbica, capacidad anaeróbica, higiene y alimentación.

La aptitud física es la condición natural o innata que tiene un individuo para realizar actividades físicas en forma eficiente; y está determinada por la capacidad motora y la capacidad física. La capacidad motora se relaciona con habilidades y destrezas generales y específicas. Las generales son actividades dirigidas a: lanzar, atrapar, saltar, trepar, girar, rodar, reptar, escalar, trotar, correr, entre otras; y las actividades específicas se ejecutan a través de: dribles, lanzamientos, voleos, manchetas, saques, apoyos invertidos, volteretas, entre otras. La capacidad física se relaciona con las valencias físicas o cualidades fisiológicas, como la resistencia aeróbica (resistencia general), la resistencia anaeróbica o potencia anaeróbica (resistencia muscular, potencia muscular, fuerza muscular y velocidad) y la flexibilidad (movilidad articular y elongación muscular).

El ritmo corporal tiene como objetivo el aprovechar los efectos estimuladores de la música en relación al movimiento corporal. Este bloque está conformado por los siguientes contenidos: rondas tradicionales, populares y modernas, balies tradicionales, populares y modernos, y danzas tradicionales populares y modernas. En la I Etapa de Educación Básica solo se desarrollan contenidos con rondas y bailes.

La vida al aire libre promueve la formación integral del ser humano por medio de actividades motrices realizadas en ambientes naturales. La intencionalidad de los contenidos en este bloque es la de fomentar a que el niño/a asuma una actitud de respeto, conservación y mantenimiento del medio ambiente; descubra y aprenda en conjunto los aportes de las actividades al aire libre como facilitadoras de aprendizajes conectados, que requieren una relación vital y significativa con el medio natural, a objeto de que el educando valore la actividad física, la 
recreación, la interacción personal con la naturaleza y el buen uso del tiempo libre.

La expresión y comunicación corporal reconoce el cuerpo y el movimiento como ejes básicos de la acción educativa. El cuerpo tiene, además del valor funcional, un carácter social que se deriva de sus propiedades expresivas y del significado que los otros le atribuyen en situaciones de interacción. La expresión corporal se encuentra implícita en toda acción desarrollada por el cuerpo. Este bloque está constituido por los siguientes contenidos: mímicas y pantomimas, dramatizaciones, bailes, danza.

\section{Investigación empírica sobre el currículum de la EF}

En base a lo expuesto se plantean las siguientes interrogantes:

1. ¿Existe un proceso de planificación previo por parte de los docentes para la organización de la enseñanza de la Educación Física?

2. ¿Cuáles son los elementos de la planificación presentes en la práctica escolar de la Educación Física en las Escuelas Bolivarianas?

3. ¿Cuál es la relación entre práctica escolar y los diseños curriculares, desde la perspectiva de los docentes de Educación Física?

4. ¿En qué medida las prácticas escolares responden a las finalidades del currículum como intención?

En definitiva, este trabajo pretende reconocer las debilidades y fortalezas en la implementación de los diseños curriculares de Educación Física en relación a los docentes que laboran en las Escuelas Bolivarianas, y sobre esas bases proponer mejoras para contribuir con su formación permanente en beneficio de los educandos.

\section{Objetivos}

\section{General}

Evaluar el estado actual del currículum de Educación Física en la escuela bolivariana, y su puesta en práctica desde la perspectiva de los docentes. Bases para la formulación de una propuesta de mejora.

Objetivos específicos

1. Describir los elementos de la planificación presentes en la práctica escolar de la Educación Física en las Escuelas Bolivarianas.

2. Conocer la relación entre práctica escolar y los diseños curriculares, desde la perspectiva de los docentes de Educación Física.

3. Proponer acciones de mejora para la implementación del diseño curricular de Educación Física.

\section{Metodología, instrumentos y muestra}

Se trata de una investigación de campo, de carácter descriptivo. La investigación se inscribe en las llamadas ex-postfacto o no experimental, entendida por Hernández, Fernández y Baptista (1998: 189) «como la observación sin posibilidades de influencia del investigador sobre la situación considerada». Se siguió también el modelo que realizan Albarracín y Moreno (2012) en clases de Secundaria con EF o de Pieron (2012) que refiere metodologías de estudio de la Didáctica de la EF. La investigación se apoya en un modelo mixto y ex-postfacto. Los motivos por los que se selecciono esta metodología es que se trabaja con hechos ya sucedidos, la intervención directa no es posible, se describe lo existente, se analiza y se hacen propuestas para la mejora. El diseño completo es el que se presenta a continuación: 
Tabla 1. Diseño de la investigación

Educación fisica, currículum y práctica solar

\begin{tabular}{|c|c|}
\hline Objetivo general & $\begin{array}{l}\text { Evaluar el estado actual del currículum de Educación Física en la Escuela } \\
\text { Bolivariana, con respecto a su administración desde la perspectiva de los } \\
\text { docentes. Bases para la formulación de una propuesta de mejora. }\end{array}$ \\
\hline \multirow[t]{5}{*}{ Objetivos específicos } & $\begin{array}{l}\text { Describir las características de los docentes de EF al finalizar su formación } \\
\text { profesional. }\end{array}$ \\
\hline & $\begin{array}{l}\text { Describir los elementos de la planificación presentes en la práctica escolar } \\
\text { de la EF en las Escuelas Bolivarianas. }\end{array}$ \\
\hline & $\begin{array}{l}\text { Describir los elementos de la planificación presentes en la práctica de la EF } \\
\text { en las Escuelas Bolivarianas. }\end{array}$ \\
\hline & $\begin{array}{l}\text { Conocer la relación entre la práctica escolar y los diseños curriculares } \\
\text { desde la perspectiva de los docentes de EF. }\end{array}$ \\
\hline & Proponer acciones de mejora para la implementación del diseño curricular de EF. \\
\hline \multirow[t]{7}{*}{ Para responder a... } & ¿Cuáles son las características de los docentes de EF al finalizar su formación? \\
\hline & ¿Existe un proceso de planificación previo por parte de los docentes \\
\hline & para la organización de la enseñanza de la EF? \\
\hline & $\begin{array}{l}\text { ¿Cuáles son los elementos de planificación presentes en la práctica escolar } \\
\text { de estos docentes? }\end{array}$ \\
\hline & $\begin{array}{l}\text { ¿Cuál es la relación existente entre la práctica escolar y los diseños curriculares } \\
\text { desde la visión de los docentes de EF? }\end{array}$ \\
\hline & ¿En qué medida las prácticas escolares responden a las finalidades \\
\hline & del currículum como intención? \\
\hline \multirow[t]{6}{*}{ Metodología } & Fase teórica de revisión de la literatura. \\
\hline & Selección y justificación del modelo de investigación descriptivo-explicativo. \\
\hline & El contexto: las Escuelas Bolivarianas del Municipio de Girardot. \\
\hline & Selección muestral y diseño de instrumentos. \\
\hline & Aplicación de dos instrumentos: cuestionarios y grupos de discusión. \\
\hline & Análisis de resultados, discusión y conclusiones. \\
\hline \multirow[t]{3}{*}{ Fundamentado en... } & Procesos de desarrollo curricular y profesional. \\
\hline & Visión desde la organización escolar y su incidencia. \\
\hline & Detección de fortalezas, debilidades y áreas de mejora. \\
\hline
\end{tabular}

Para conseguir una visión descriptivo-interpretativa del desarrollo curricular de las clases de Educación Física y su vinculación con las propuestas curriculares en el municipio de Girardot para ayudar a tomar decisiones administrativas, curriculares y de gestión.

Fuente: adaptado de Cantón (2004).

Se puede decir que el diseño tiene un desarrollo «emergente» por las veces que se debe buscar y recomponer lo previsto, en síntesis el resultado es el que mostramos resumido en el gráfico al incorporar hallazgos no previstos en el diseño de la investigación y exige una alta capacidad interpretativa del investigador (Cantón, 2004, 2000, 2009). El diseño emergente del que hablan Lincoln y Guba (1994) es necesario para toda investigación cualitativa, porque refleja en la investigación la realidad y los puntos de vista de los participantes, en este orden de ideas De 
Miguel (2000: 47) siguiere tenerse en cuenta los llamados por decálogo del investigador: «olfato, experiencia, observación, interés, espíritu crítico, independencia, movilidad, continuidad, creatividad y claridad».

En la Tabla 2 se presentan los objetivos planteados y el tratamiento de los mismos a partir de los diferentes momentos planteados en el modelo de evaluación CIPP.

Los instrumentos que se utilizaron para la recogida de datos en esta investigación fueron:

- El cuestionario.

- Grupos de discusión.

Para Ander-Egg (2000: 124), el cuestionario contiene «aspectos del fenómeno que se consideran esenciales, permite además aislar ciertos problemas que nos interesan, principalmente reduce la realidad a cierto número de datos esenciales y precisa el objeto de estudio». A través de este se obtuvieron los datos cuantitativos de la investigación. Sin embargo, el modelo cualitativo nos permitió llegar a la comprensión del fenómeno a partir de las experiencias de los propios autores, por lo que utilizamos la técnica de grupos de discusión. Según Valles (1999), los grupos de discusión y la encuesta son una articulación apropiada y documentada, según este autor la encuesta pueden adoptar funciones diferentes. La complementariedad de los grupos de discusión con el cuestionario en nuestra investigación persigue la ilustración y confirmación de los resultados cuantitativos, asimismo, la clarificación y elaboración de los resultados de la encuesta que podrían haberse considerado contradictorios de no haber contado con la información cualitativa y la sugerencia de dimensiones y variables explicativas no anticipadas en la fase de diseño. Estas fueron las categorías del cuestionario (tabla 3).

Tabla 2. Correspondencia entre objetivos y tipo de evaluación propuestos en la investigación

Objetivos

Evaluación de contexto

Evaluación de entrada

Evaluación de proceso

Evaluación de producto

Evaluación de contexto, Evaluación de entrada, Evaluación de proceso y Evaluación de producto
Fase de la evaluación

Describir las características de los docentes de Educación Física al finalizar su formación profesional. Caracterizar la aplicación del currículum en la planificación de la Educación Física en las Escuelas Bolivarianas.

Describir los elementos de la planificación presentes en la práctica escolar de La Educación Física en las Escuelas Bolivarianas.

Conocer la relación entre práctica escolar y los diseños curriculares, desde la perspectiva de los docentes de Educación Física.

Proponer acciones de mejora para la implementación del diseño curricular de Educación Física. 
Tabla 3. Correspondencia entre evaluación, categorías y subcategorías e ítems en el cuestionario

\begin{tabular}{|c|c|c|c|}
\hline Categorías & Subcategorías & Indicadores & Ítems \\
\hline \multirow{7}{*}{$\begin{array}{l}\text { Evaluación } \\
\text { de contexto }\end{array}$} & \multirow[t]{7}{*}{ Perfil profesional } & $\underline{\text { Sexo }}$ & 1 \\
\hline & & Edad & 2 \\
\hline & & Experiencia laboral & 3 \\
\hline & & Título & 4 \\
\hline & & Otros títulos & 5 \\
\hline & & Tipo de jornada en la que trabaja & 6 \\
\hline & & Horas semanales de docencia & 7 \\
\hline \multirow{3}{*}{$\begin{array}{l}\text { Evaluación } \\
\text { de entrada }\end{array}$} & \multirow{2}{*}{$\begin{array}{l}\text { Conocimiento } \\
\text { del currículum }\end{array}$} & Documentos oficiales & 8 \\
\hline & & Diseños curriculares & $9,10,11,12$ \\
\hline & $\begin{array}{l}\text { Conocimiento } \\
\text { de la planificación }\end{array}$ & $\begin{array}{l}\text { Utilización de los diseños en la planificación } \\
\text { de las clases }\end{array}$ & $13,14,15,16,17,18$ \\
\hline \multirow[t]{4}{*}{$\begin{array}{l}\text { Evaluación } \\
\text { de proceso }\end{array}$} & \multirow[t]{4}{*}{ Implementación } & Secuencia y continuidad & $\begin{array}{l}19,20,21,22,23 \\
24\end{array}$ \\
\hline & & Evaluación de aprendizaje & $25,26,27,28,29$ \\
\hline & & Actividades & $\begin{array}{l}30,31,32,33,34 \\
35,36\end{array}$ \\
\hline & & Recursos & $37,38,39,40,41$ \\
\hline \multirow[t]{6}{*}{$\begin{array}{l}\text { Evaluación } \\
\text { de producto }\end{array}$} & \multirow[t]{6}{*}{ Pertinencia } & $\begin{array}{l}\text { Vinculación de los diseños curriculares/ } \\
\text { prácticas docentes }\end{array}$ & $42,43,44,45,46$ \\
\hline & & Actividades de aprendizajes: vida al aire libre & $47,48,49,50,51$ \\
\hline & & $\begin{array}{l}\text { Actividades de aprendizajes: expresión } \\
\text { y comunicación }\end{array}$ & $52,53,54,55$ \\
\hline & & Actividades de aprendizajes: juegos motrices & $\begin{array}{l}56,57,58,59,60 \\
61\end{array}$ \\
\hline & & Actividades de aprendizajes: aptitud física & $62,63,64,65,66$ \\
\hline & & Actividades de aprendizajes: ritmo corporal & $67,68,69,70,71$ \\
\hline
\end{tabular}

El cuestionario fue validado por 6 expertos: tres universitarios especialistas en EF y tres docentes de Educación Física. Los criterios de validación fueron pertinencia, relevancia y univocidad. Cuando dos o más discrepaban en un ítem se reformulaba o se eliminaba en función de la validación obtenida. Del primer cuestionario de 90 ítems se llegó así a un cuestionario final de 71 ítems.

Los grupos de discusión fueron 3 en los que intervinieron 15 profesionales de la EF moderados por la investigadora teniendo como eje las categorías del cuestionario señaladas anteriormente.

La muestra la constituyeron 65 docentes de EF de Educación Primaria del estado de Aragua y fue seleccionada al azar por medio de sorteo entre una población de 350 docentes de más de 100 escuelas del municipio de Aragua.

Se realizó un cronograma de trabajo para la recogida de datos que duró un año. Finalmente, para codificar los datos se siguió el procedimiento de Taylor y Bodgan (1992). 


\section{Resultados}

Damos algunos de los datos en el gráfico y posteriormente señalamos únicamente la interpretación de los resultados ya que sería muy prolijo representar todos los datos obtenidos. En primer lugar, los datos de la muestra y a continuación los de entrada, proceso y producto.

En cuanto al género, el 32,31\% de los docentes de Educación Física de las Escuelas Bolivarianas del municipio Girardot del estado Aragua son mujeres y el $67,69 \%$ son hombres. Se

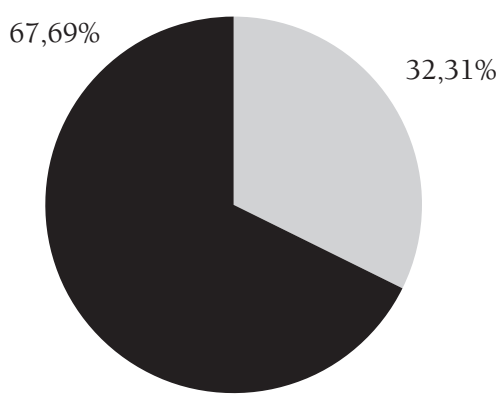

Femenino

Masculino

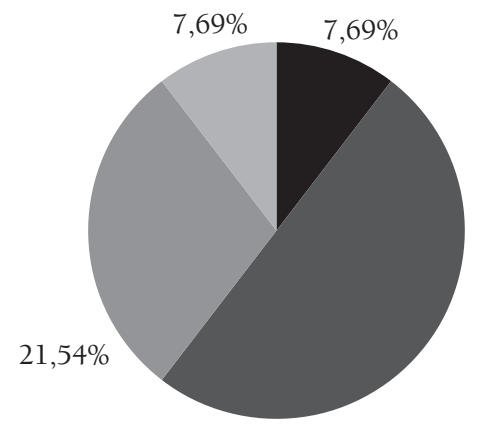

$36,92 \%$

20-30

$31-40$

$41-50$

Más de 50 podría inferir que esta tendencia corresponde a las características de la especialidad, o a las pruebas especiales que presentan los aspirantes para ingresar a la carrera de educación como requisito indispensable en el área de Educación Física. El 63,08\% de los docentes tienen edades comprendidas entre 31/40 años, un 7,69\% entre $20 / 30$ años, un $21,54 \%$ entre $41 / 50$ años y un $7,69 \%$ tienen más de 51 años de edad. Por lo que se puede concluir que el $84,62 \%$ de los docentes de Educación Física del municipio Girardot es una población relativamente joven, ya que se encuentran en edades comprendidas entre los 31 y los 50 años de edad.

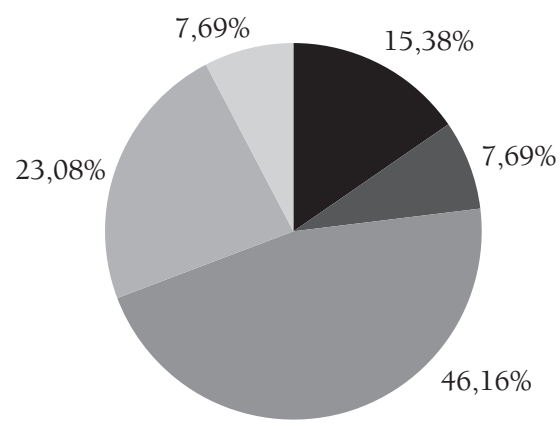

Menos de 5 años Entre 5 y 10 años Entre 11 y 15 años Entre 16 y 20 años 21 años y más

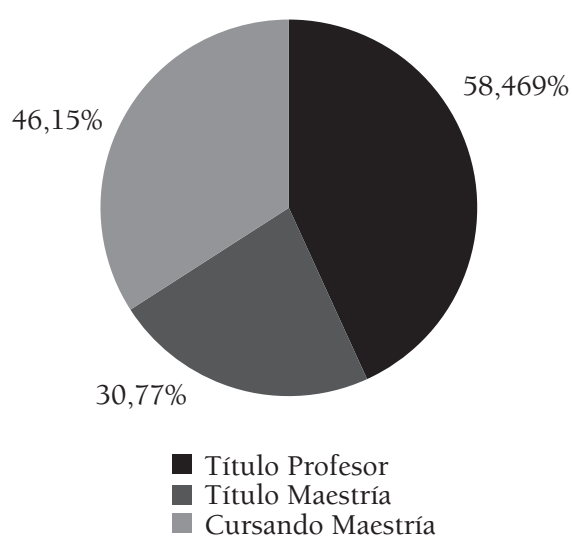


En la experiencia docente se visualiza que la mayor cantidad de profesores está entre 11 y 15 años con el 46,15\%, el 23,08\% se encuentra entre 16 y 20 años, lo que significa el 69,23\% del total de los docentes que $t$ en las Escuelas Bolivarianas son profesionales con experiencia. El 58,46\% manifestó haber obtenido el título de Maestría en Educación y el 10,77\% está cursando actualmente, por lo que el $41,54 \%$ de estos profesionales están profundizando en su campo del saber, ampliando y desarrollando sus conocimientos para actuar como investigadores para solucionar problemas puntuales que se le presenten en su profesión. Con respecto al título del profesor, son docentes egresados de la universidad pedagógica experimental libertador y los que no están graduados cursan estudios de pregrado en esa institución de educación superior. El 30,77\% posee el título de Maestría.

La mayoría de las Escuelas Bolivarianas no cumple con el ordenamiento establecido en atención al horario de jornada de trabajo establecido para dichas escuelas debido a la falta de infraestructura para funcionar en un solo turno.

\section{Resultados de la evaluación de entrada}

Los datos tanto cualitativos como cuantitativos muestran que los docentes no tienen conocimiento claro acerca de los documentos emanados por el Ministerio del Poder Popular para la educación bolivariana. Entre las causas que los propios profesores admiten para que esto suceda así, se deben a que son:

«unos materiales elaborados al margen del colectivo del profesorado», son «unos documentos con un léxico de gran complejidad», a la vez que han sido «informados muy poco sobre los materiales y lo que supone la reforma...». Lo podemos apreciar en frases tales como: «encuentro ciertas dificultades en la asimilación de todos estos términos: proyecto de comunitario, proyecto educativo, proyecto de aprendizaje..., a veces me pierdo a mitad de camino y en varias ocasiones he intentado llevármelos a casa para trabajarlos y no hay manera de poderlo hacer...» (Gl: 1).

«Se supone que todos lo conocemos pero no sabemos cómo se lleva a cabo y si realmente se da» (G2: 1).

Lo anterior indica que existe un conocimiento superficial. Es decir poca profundidad en cuanto al conocimiento de los proyectos comunitarios, proyectos educativos y proyectos de aprendizaje, entre otros, para trabajarlos en cada nivel y modalidad del sistema educativo. Por lo que se infiere un accionar profesional basado en la experiencia sin referencia teórica. La siguiente opinión lo describe claramente:

«se hacen los proyectos curriculares porque se nos exige, e incluso, con fecha concreta de presentación y todo, pero en realidad, en la práctica, aún no se está llevando a cabo, o sea, solo se hace por cumplir unos "papeles"; y a veces ejecutamos algo y no sabíamos existía como requisito en los documentos curriculares» (Gl: 1).

Por otro lado existe contradicción en el manejo del diseño curricular de preescolar, los datos cuantitativos reflejan que existe conocimiento pero al cruzarlo con los grupos de discusión se demuestra lo contrario. En la I y II Etapa de Educación Básica se evidencia el conocimiento de los diseños lo que permite deducir que a los docentes de Educación Física les resulta fácil la concreción de las propuestas curriculares para esos niveles o modalidades del sistema educativo.

Los diseños curriculares se reconocen como propuestas generadoras para determinar acciones para llevar a cabo la práctica pedagógica. Se acepta la estructura de su organización donde se perciben los aspectos a lograr en atención al bloque de contenido a planificar.

«Todos los docentes debemos tener nuestros diseños curriculares para regirnos en 
función de ellos. Sin embargo, yo los uso en esos momentos que no se que hacer para tomar ideas para planificar, los contenidos nos sirven de base para comenzar a escribir» (G2: 1).

«Los diseños curriculares nos permiten guiarnos para planificar los contenidos de manera que uno pueda ir jerarquizándolos por nivel educativo» (G2: 1).

«Por falta de tiempo copio los contenidos textualmente del programa como para cumplir con el requisito de la planificación» (G2: 2).

«Por rapidez y ligereza no analizó los contenidos y soy poco creativa e innovadora» (G2: 2).

«Debería existir un acompañamiento pedagógico donde se le comprometa al docente la selección de contenidos en atención a los proyectos de aprendizaje de los docentes de aula» (G2: 2).

Los profesores manejan superficialmente los diseños curriculares y no profundizan los que en ellos se plantea como documentos oficiales para llevar a cabo la práctica pedagógica, pareciera que tuviesen un conocimiento poco profundo según las afirmaciones expuestas.

El 61,54\% manifestó estar completamente de acuerdo en la utilidad de los bloques de contenidos. Sin embargo, el 29,23\% no estuvo de acuerdo con este planteamiento lo que lleva a inferir que estos docentes improvisan a la hora de planificar sus clases. Un 9,23\% declaró estar indeciso, la utilidad de los bloques de contenidos en la planificación como instrumento de trabajo del docente constituye en herramienta pedagógica de organización del proceso de enseñanzaaprendizaje y la revisión permanente de la práctica por parte del docente.

Los datos cuantitativos discrepan de los datos cualitativos una vez realizada la triangulación.
En el conocimiento del currículum se declara que son documentos elaborados al margen del colectivo de los docentes, infiriéndose que el accionar pedagógico está basado en la experiencia práctica sin referencia teórica. Esto en atención a los resultados obtenidos en la tendencia al desconocimiento del documento que rige a las Escuelas Bolivarianas, al diseño curricular del nivel de educación inicial.

En los diseños curriculares de la I y II Etapa de Educación Básica se considera que los docentes tienen conocimientos en esos niveles, sin embargo, a la hora de concretar la práctica pedagógica prevalece la experiencia docente para tomar decisiones curriculares, pasando por alto las concreciones propuestas en los documentos oficiales.

En relación a la utilización de los documentos curriculares en la planificación de las clases según los datos obtenidos los diseños son una guía para planificar los contenidos de manera que se puedan ordenar según el grado de dificultad en el nivel educativo. Sin embargo, en los datos cualitativos se evidencia que a la hora de planificar prevalece la falta de tiempo, ligereza, cumplir requisitos de la planificación, entre otros aspectos, y no se analizan los contenidos a desarrollar, pasando por alto su utilidad en la organización del proceso de enseñanza y de aprendizaje.

\section{Resultados de la evaluación de proceso}

Se trata ahora de conocer cómo se hacen las cosas en las clases siguiendo los aspectos curriculares señalados. Vemos únicamente lo relacionado con los juegos motrices y aptitud física en las dos dimensiones y resumimos el resto de resultados.

De acuerdo con los porcentajes que observamos en el gráfico, los docentes en un $76,92 \%$ analizan las características de los juegos motrices, para propiciar situaciones de aprendizajes 


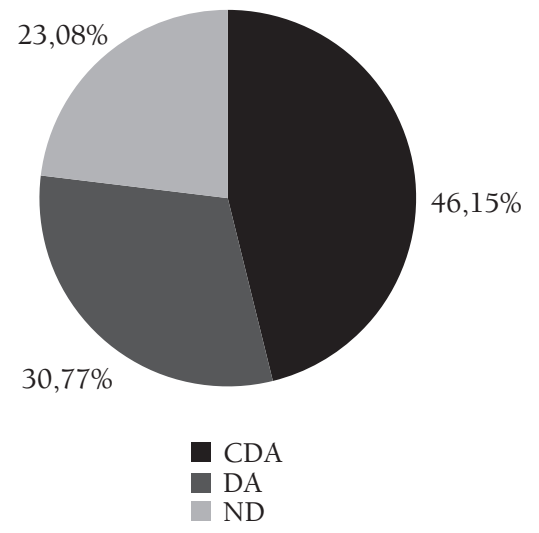

lo más educativas posible. Por lo que se infiere que en la educación básica en el área de Educación Física se fomentan los mecanismos de percepción y de decisión según su edad cronológica de los estudiantes. Asimismo, el conocimiento y cumplimiento de los principios metodológicos suponen un criterio para seleccionar actividades adecuadas para el área. Esta acotación se corrobora con el siguiente comentario al referirse a la selección de los contenidos:

«al seleccionar los juegos motrices se debe tener presente lo que el estudiante es capaz de realizar, la organización de una actividad hacia la consecución de un objetivo determinado. Asimismo se debe tener una serie de características: un objetivo que defina lo que se pretende alcanzar con la puesta en marcha de la actividad, determinadas condiciones que acompañan a la realización del objetivo, instrucciones que precisen un tipo particular de comportamiento motor o de proceso que hay que llevar a cabo y las características de los estudiantes con los que vamos a trabajar» (G2: 3).

De acuerdo con lo anterior podemos decir que de los docentes a la hora de llevar a cabo la práctica pedagógica seleccionan los juegos motrices según el grado de dificultad. El éxito pedagógico de la clase va a depender de la selección y diseño de estrategias adecuadas, que respondan a principios y

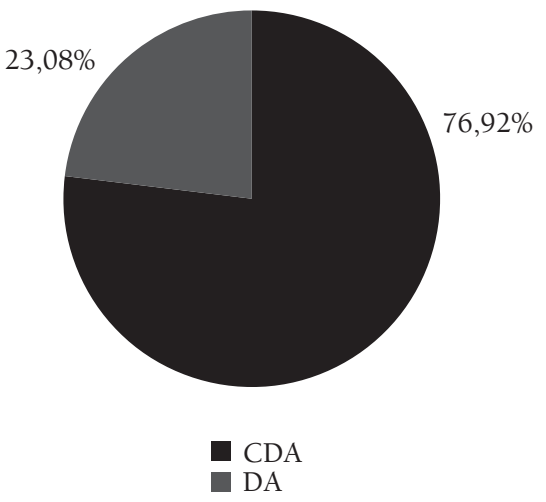

criterios fundamentales sobre lo que se va a realizar en la programación a corto, medio y largo plazo. En aptitud física el 76,92\% manifestó estar completamente de acuerdo y el 23,08\% de acuerdo en que cada actividad física propuesta implica una forma peculiar de asumirla y dependerá de las posibilidades físicas que posea cada individuo o del tipo de actividad y experiencia motriz que este tenga; así su desarrollo físico externo e interno también tendrá cambios en relación a la actividad física practicada (Rodríguez, 2011).

Los resultados generales obtenidos de la evaluación de proceso permiten caracterizar algunos elementos de la puesta en marcha del currículum en las clases de Educación Física en las Escuelas Bolivarianas del municipio Girardot del estado Aragua. Según lo planteado por Stenhouse (1984), podríamos decir que este apartado ofrece información acerca del currículum real. En relación a la continuidad y secuencia de las propuestas de las clases los resultados demuestran que en la experiencia concreta los docentes en líneas generales encuentran dificultad para realizar una práctica pedagógica sistemática y rigurosa. En los datos proyectados en el cuestionario, encontramos que los docentes manifiestan ajustar sus prácticas a lo propuesto en los documentos oficiales en atención a los criterios de selección en atención al bloque de contenido para llevar a cabo la práctica escolar, 
y que existe una tendencia elevada de no llevar a cabo la planificación integrada docente de aula y el especialista, así como tampoco se consideran las opiniones de los educandos a la hora de planificar.

El profesor de Educación Física al elaborar sus planificaciones debe tratar los diferentes bloques de contenidos de una manera global y conjunta, lo cual no coincide con la realidad según los datos obtenidos, por lo que se infiere que destacan importancia al bloque de contenido juegos motrices y aptitud física en su programación de aula, independientemente de la etapa de formación en la que se encuentran, al comprobar los datos obtenidos en el cuestionario en relación a las actividades planificadas, ya que la tendencia a nivel porcentual es elevada. Por lo que se considera evidente, que en la planificación de los contenidos en una programación de aula en el área de Educación Física, pareciera que depende en mayor medida a los contenidos que dominan los docentes.

En la evaluación de los aprendizajes en los datos obtenidos en el cuestionario nuevamente nos encontramos que mayoritariamente el profesorado reconoce evaluar para conocer los conocimientos previos, proceso o avance en los estudiantes. Sin embargo, esta evaluación para un porcentaje menor de docentes no se realiza utilizando instrumentos de evaluación que permitan arribar a valoraciones objetivas y fundamentadas en criterios consistentes y objetivos. Esta falta de correspondencia entre una información y otra le añadimos la ausencia de datos cualitativos en relación con el tema, por lo que se infiere que las prácticas de evaluación son de escasa firmeza pedagógica.

Según los datos obtenidos en las instituciones educativas no se cuenta con los recursos o medios, así como tampoco con los espacios adecuados para llevar a cabo la práctica escolar. Lo que trae como consecuencia que los docentes no puedan acceder eficazmente a los contenidos y proponer actividades y espacios de participación para la necesaria contextualización y transferencia de los conocimientos en los educandos.

Por otro lado, al planificar las actividades, se debe tener en cuenta que todos aprendemos de maneras diversas, y que cada estudiante como ser único tiene necesidades particulares. Por ello, debemos enfocar las fortalezas del niño/a con la idea de propiciar la adquisición de conocimiento para que el estudiante pueda construir aprendizajes de forma natural, con o sin ayuda del docente.

\section{Resultados de la evaluación de producto}

En este apartado se presentan las percepciones que tienen los docentes en relación a su práctica y lo que expresan los documentos curriculares. Primero presentamos lo que piensan acerca
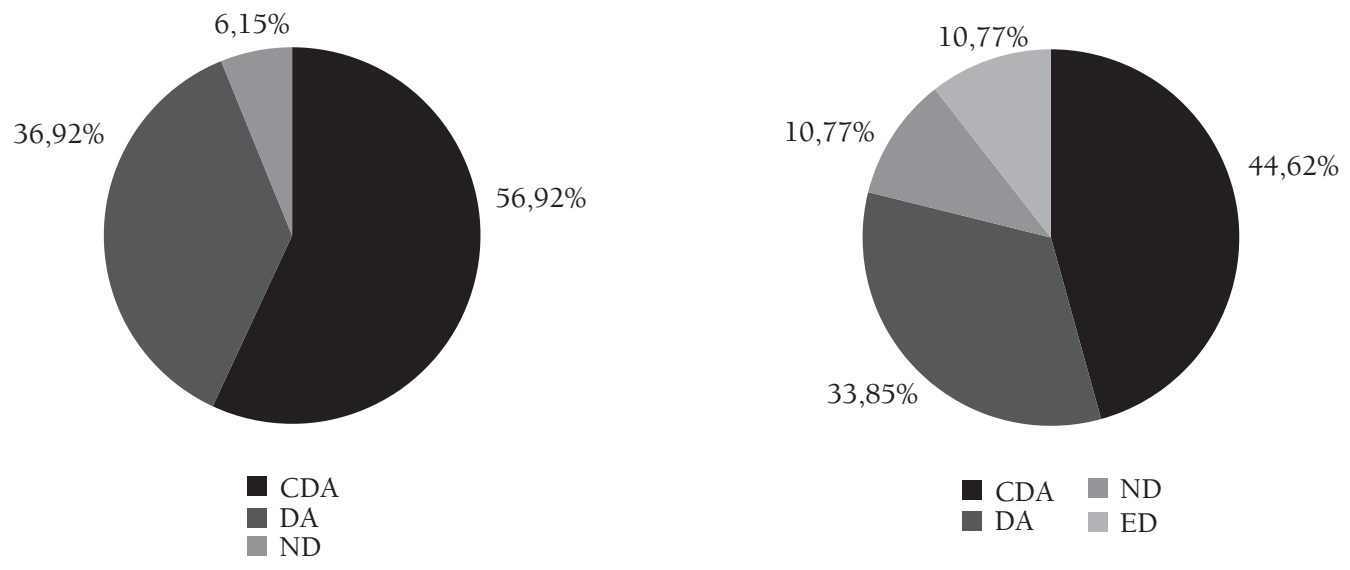
de la posibilidad de concretar estas ideas en la práctica profesional, luego cuál es su actitud acerca de la incidencia de los actores escolares en la definición de la práctica cotidiana y, finalmente, las posturas en relación a los contenidos propuestos para el área de Educación Física.

En el ítem n ${ }^{\circ} 42$, la mayoría de los docentes manifestó que desde la asignatura de Educación Física se deben instruir ciudadanos que participen en la transmisión de valores que se promulgan en la sociedad. Esto implica que desde las instituciones educativas se debe fomentar propuestas y programas que difundan la educación en valores. Sin embargo, el 6,15\% manifestó estar indeciso con este planteamiento. Lo que se corrobora con los datos cualitativos:

«El ciudadano y la sociedad que queremos formar, sobre la realidad que nos envuelve, va más allá de la discusión sobre doctrinas sociológicas o pedagógicas de autores particulares, sería posible diseñar un sistema educativo que sea espacio de realización de las capacidades de cada individuo y de aprendizaje práctico de la vida social solidaria, en un ambiente democrático en la toma de decisiones» (G3: 1).

«Toda educación se debe ajustar a los principios y metas constitucionales y de bien común. La educación, al ser un bien público, debe proporcionar la participación de la sociedad y generar conciencia ciudadana» (G3: 1).

«El derecho a la educación es innato al niño y produce en correspondencia unas obligaciones y responsabilidades en los padres, en la sociedad y en el Estado» (G3: 1).

El ítem $n^{\circ} 43$ arrojó que la mayoría de los docentes consideran que los estudiantes deben ser protagonistas de su aprendizaje, tomando del ambiente los elementos de su estructura cognitiva que sea capaz de asimilar, y así construir su propio conocimiento logrando alcanzar su aprendizaje significativo. Estos datos se reafirman con los datos cualitativos.
«En el quehacer profesional nosotros como docentes debemos fomentar que todo estudiante a partir de sus experiencias pueda construir su propio conocimiento, y se dé cuenta de sus debilidades y potencialidades» (G3: 2).

«El trabajo docente es crear un lenguaje que logre establecer las conexiones necesarias para que el estudiante vincule el conocimiento de la calle con el conocimiento científico, de manera tal que el aprendizaje se haga consciente y por consiguiente significativo para los ellos» (G3: 2).

La evaluación producto en resumen presenta las percepciones que tienen los docentes en relación a su práctica y lo que expresan los documentos curriculares, se evidenció que tienen relación con los resultados de los apartados anteriores. Asimismo los datos cuantitativos en muchos de los casos se respaldan con los cualitativos obtenidos en los grupos de discusión.

En relación a la vinculación de los diseños curriculares/prácticas docentes desde las instituciones educativas, la Educación Física está sustentada en la reflexión sobre los fines de la educación, el estudiante construye su propio conocimiento, los miembros de la comunidad no asisten a las actividades que se planifican en la institución, y en consecuencia no participan en las tomas de decisiones en busca de soluciones para la puesta en práctica de proyectos. Por otro lado, las innovaciones pedagógicas para la puesta en práctica de nuevas ideas o estrategias en beneficio de la institución se imponen.

Los juegos motrices y la aptitud física son los boques de contenidos más utilizados en el proceso de enseñanza y aprendizaje del área, existe una marcada tendencia en los datos cuantitativos y cualitativos que la planificación de las clases está subordinada a esta afirmación. En tal sentido, la continuidad de las experiencias está relacionada con la preparación de los estudiantes en actividades deportivas y de mejora de las condiciones 
físicas, como si estas fuesen el fin del área establecido en los diseños curriculares.

Pareciera que la Educación Física cuenta con una frágil consideración en las propuestas de enseñanza, debido a que en las actividades al aire libre, a pesar que los profesores manifestaron están completamente de acuerdo y conformes con que estas brindan a los estudiantes la posibilidad de disfrutar de la naturaleza, en los datos obtenidos se infiere que los docentes en la implementación de sus clases incorporan muy pocas actividades dirigidas a este bloque de contenido (Cantón Mayo y Pino Juste, 2011). Al igual que actividades de aprendizaje relacionadas con el ritmo corporal y expresión y comunicación corporal, en los documentos curriculares el área de Educación Física debe favorecer el desarrollo e incremento de la habilidades motrices, ejercitar movimientos coordinados, que le permitan al estudiante el desarrollo de su personalidad, expresarse y proyectarse como persona, por medio de diferentes actividades físicas donde se involucren estos contenidos.

Es viable que el planteamiento anterior sea consecuencia de la formación de pregrado de los docentes en Educación Física, lo que significa que su egreso como profesionales del área no se caracteriza bajo la concepción y lineamientos establecidos en los diseños curriculares, y, por otro lado, su proceso de formación permanente este orientado hacia el área deportiva sin ningún tipo relación con la enseñanza de la Educación Física en las Escuelas Bolivarianas.

Por otro lado, en los resultados obtenidos en las preguntas abiertas del cuestionario, la mayoría de los docentes considera que los diseños curriculares son interesantes, lo que confirma que el actual diseño tiene una valoración positiva para ellos, y expresaron que consideraban importante la planificación integrada, la integración escuelacomunidad, la flexibilidad del currículum, pero les dificultaba llevarlo a la práctica debido a la falta de acompañamiento pedagógico, insuficiente formación permanente e insuficiente recursos materiales. Entre las ideas que les parecen menos interesantes está el desarrollo de bailes en las clases, incorporar pantomimas e incorporar danzas.

En relación a la pregunta, además del voleibol, baloncesto y fútbol sala, qué otro(s) deporte(s) planifica, respondieron: kickimbol, fútbol de campo, béisbol. El docente es un profesional, su acervo pedagógico le permite contener un conjunto de conocimientos, competencias didácticas, habilidades intelectuales, actitudes éticas y valores que le conceden, a partir de su propia experiencia motriz, entender y comprender al niño en todo su proceso de desarrollo, biológico, motor, social, psicológico y moral, a fin de adecuarse a las condiciones del medio y del entorno de la escuela ya que el deporte a nivel escolar es una vía para contribuir al desarrollo integral de los estudiantes.

Finalmente, entre los temas de formación permanente se sugiere enseñar a optimizar la administración de los diseños curriculares en las prácticas educativas; atender las necesidades de adaptación a los procesos de cambio en el ejercicio profesional en atención a aspectos pedagógicos; y en tercer lugar, se hace mención a la formación de los docentes en y para los deportes (Albarracín y Moreno, 2012; Rodríguez, 2012).

\section{Referencias bibliográficas}

Albarracín Pérez, A. y Moreno Murcia, J. A. (2012). Análisis de contenidos y actividades desarrolladas en las clases de actividades acuáticas: una propuesta para la Enseñanza Secundaria. En Apunts, de segundo trimestre, $\mathrm{n}^{\circ}$ 108, pp. 32-43.

Ander-EgG, E. (2000). Técnicas de investigación social. Argentina: Editorial humanista.

Apple, M. (1983). Ideología y currículo. Madrid: Akal. 
Bernstein, B. (2000). Hacia una sociología del discurso pedagógico. Santafé de Bogotá: Magisterio.

CANTÓn MAYo, I. (2000). La calidad total: evolución, concepto e implementación. En I. Cantón (coord.), Las organizaciones escolares: hacia nuevos modelos. Buenos Aires: Fundec.

Cantón Mayo, I. (2004). Evaluación de los Planes de Mejora en los centros públicos de Castilla y León. Universidad de León.

CAntón Mayo, I. (2009). Modelo sistémico de Evaluación de Planes de Mejora. Universidad de León. Cantón Mayo, I. y Pino Juste, M. (coords.) (2011). Diseño y desarrollo del currículum. Madrid: Alianza.

Castro F. y Lira, H. (2004). Currículum y evaluación texto guía. Chile: Edición Primera.

Coll, C. (1989). Lo básico en la educación básica. Reflexiones en torno a la revisión y actualización del currículo de la educación básica. [Revista en Línea] Disponible: http: //redie.uabc.mx/vol8nol/ contenido-coll.html [Consulta: 2010, enero 07]

Comisión Nacional de Currículum (1997). Vicerrectorado Académico de la Universidad de Carabobo. Principios para la transformación y modernización académico curricular en la educación superior venezolana.

Consejo Internacional para la Salud, Educación Física, Recreación, Deporte y Danza (ICHPER SD) (2001). Comité Ejecutivo.

De Miguel, M. (2000). La evaluación de programas sociales: fundamentos y enfoques teóricos. En Revista de Investigación Educativa, vol. 18, n² 2.

Espinoza, A. (2001). Pasos y tesis curriculares. España.

GAGNÉ, E. (1990). La planificación de la enseñanza: sus principios. México: Trillas.

Gervilla, A. (2006). El curriculum: fundamentación y modelos. El modelo ecológico. Málaga: Innovare.

Gimeno, J. (1995). El curriculum: una reflexión sobre la práctica. Madrid: Morata. $3^{2}$ edición.

GiRoux, H. (1990). Los profesores como intelectuales. Madrid: Paídos/MEC.

Habermas, J. (1998). Teoría de la acción comunicativa. Madrid: Taurus.

KeMmis, S. (1996). El currículum: más allá de la teoría de la reproducción. Madrid: Morata.

Ley Orgánica de Educación (2009). Gaceta Oficial de la República Bolivariana de Venezuela (Extraordinario). Caracas: Venezuela.

McTaggart, R y Singh, M. (1986). New Directions in Actions Research. En curriculum perspectives, vol. $6, \mathrm{n}^{\mathrm{o}} 2$.

Ministerio de Educación (1992). Programa de Estudio y Manual del Docente. Asignatura Educación Física y Deporte. Edición: Especial. Caracas: Venezuela.

Ministerio de Educación (1997). Currículo Básico Nacional Programa de estudio del Área de Educación Física de la II etapa de Educación Básica Edición: Especial. Caracas: Venezuela.

Ministerio de Educación, Cultura y Deportes (1999). Resolución N 179. Creación de Escuelas Bolivarianas.

Ministerio de Educación, Cultura y Deportes (2000). Versión preliminar del Proyecto Educativo Nacional. Caracas: Autor.

Ministerio de Educación y Deportes (2004). La educación Bolivariana. Políticas, programas y acciones «cumpliendo las metas del milenio». [Documento en línea]. http: //www.me.gob.ve/modules. php? name=Content\&pa=showpage\&pid=221 [Consulta: 2006, Octubre 21] .

Ministerio de Educación y Deportes (2005). Educación Inicial Bases Curriculares [ Documento en línea]. http: //www.edumedia.org.ve/inicial/pdf1.pdf. [Consulta: 2007, Febrero 21].

Ministerio del Poder Popular para la Educación (2007). Currículo Nacional Bolivariano. Caracas: Autor.

Pieron, M. (2012). Metodología de investigación en pedagogía de la actividad física y del deporte: progresos del conocimiento (Parte I) Revista de educación física: Renovar la teoría y práctica, $\mathrm{n}^{\circ} 126,2012$, pp. 5-14.

PINAR, W. (1999). «Not burdens: Breakthroughs». Curriculum Inquiry: 29, 365-367. 
Popkewitz, T. (1994). Sociología política de las reformas educativas. El poder en la enseñanza, la formación del profesorado y la investigación. Madrid: Morata.

Rodríguez, N. B. (2011). Educación física y formación del profesorado en Latinoamérica. En ÄGO$R A, n^{\circ} 13$ de septiembre, Monográfico.

Rodríguez, R. (1997). Transversalidad curricular y democracia en el contexto europeo. Revista de educación no 309. Madrid: Centro de publicaciones del Ministerio de Educación y Cultura.

Román Pérez, M. y Díez López, E. (2003). Aprendizaje y currículum. Diseños curriculares aplicados.

( $6^{\circ}$ edición, $1^{\circ}$ reimpresión) Novedades Educativas. Buenos Aires.

SAYLOR, J. (1970). Planteamiento del currículum en la escuela moderna. Buenos Aires: Troquel.

Stenhouse, L. (1984). Investigación y desarrollo del currículum. Madrid: Morata.

TABA, H. (1991). Curriculum development: theory and practice [La elaboración del currículo], (Alpert, Rosa trad.). Argentina: Editorial Troquel, S.A. (el original fue publicado en 1962).

TAYLOR, S. J. y BOGDAN, R. (1992). Introducción a los métodos cualitativos en investigación. La búsqueda de los significados. Barcelona: Paidós.

TJeERdsma Blankenship, B. (2012). Una comparación de las perspectivas del profesor y del estudiante de las tareas y del feedback (Parte I). Revista de educación física: Renovar la teoría y práctica, $\mathrm{n}^{\circ} 125$, 2012 , pp. 17-20.

VAlLÉs M, (1999). Técnicas cualitativas de investigación social: reflexión metodológica y práctica profesional. Madrid: Síntesis.

WheEler, D. (1993). El desarrollo del currículum escolar. 4ª edición. Madrid: Santillana.

Zabalza, M. (1991). Diseño y desarrollo curricular. Madrid: Narcea.

\section{Abstract}

From Theory to Practice: The Physical Education Curriculum In Primary Schools In Venezuela

INTRODUCTION. The aim of this study is to verify the similarity or find out the discrepancy between the prescribed curriculum and the real curriculum taught in the classroom in the subject of Physical Education in the Primary Schools of Venezuela. For this purpose, the topic is revisited from a general curricular perspective that includes three common approaches: the technical, the interpretive and the hermeutical or critical. The proposals and normative Curricular Plans of the state of Venezuela for Primary Education are then reviewed and described in terms of these three approaches, with a focus on the technical approach. The METHODS used was an evaluation of the programs using Stuflebeam's CIPP model. We also administered questionnaires to a representative sample of teachers and complemented these with qualitative instruments such as observation and interviews. The study evaluated elements such as physical aptitude, body rhythm, and outdoor activity at three different stages - the planning of teaching, the relationship between classroom practice and the prescribed Curricular Plan, and proposals for improvement. RESULTS. The research findings indicate that there is a significant disparity between what is prescribed and what is taught, and that this gap varies depending on the the category analyzed within the curriculum of Physical Education. DISCUSSION. This article is relevant in that it reveals the need to bring the reality of the classrooms closer to what is officially regulated. The representativeness of the sample would be greater if this study were reproduced in other states of Venezuela.

Key words: Prescribed Curriculum, Realized Curriculum, Evaluation of Programs, Primary Education, Motor Skill Games, Physical Aptitude, Body Rhythm, Outdoor Activities. 
Résumé

De la théorie à la pratique: le programme de l'éducation physique dans les écoles primaires du Venezuela

L'OBJECTIF de cette recherche est de connaître une homogénéité ou la divergence entre le curriculum prescrit et le curriculum réel de salle dans la matière d'Education Physique dans les Écoles Primaires de la Venezuela. Pour cela une révision du sujet est réalisée depuis la perspective curricular générale en englobant les trois courants habituels : technique, interprétative ou hermeneútica et une critique. Tout de suite les propositions sont révisées et ils décrivent et les Dessins Curriculares normatifs dans l'État de la Venezuela pour l'Enseignement primaire, qui est encadré dans les trois dimensions citées, avec supériorité de la perspective technique. La MÉTHODE DE RECHERCHE a été descriptive et d'une évaluation de programmes avec le modèle CIPP de Stuflebeam et on a utilisé les questionnaires qui se sont appliqués à un échantillon représentatif d'enseignants complétés avec des instruments qualitatifs comme l'observation et l'interview. On a évalué aspects comme l'aptitude physique, le rythme corporel, la vie en plein air et l'expression et la communication corporelle dans trois niveaux : la planification de l'enseignement, de la relation entre la pratique de salle et le Dessin Curricular prescrit et la proposition d'actions d'améliorations. LES RÉSULTATS ET LA DISCUSSION montrent que la distance entre le prescrit et le réalisé est ample et différente pour les catégories distinctes analysées, à l'intérieur du curriculum d'Education Physique. Larticle est éminent pour montrer la nécessité de rapprocher la réalité des salles de classe à la réglementation. La représentativité de l'échantillon serait plus grande si la recherche était répliquée dans d'autres états de Venezuela.

Mots Clés: curriculum prescrit, curriculum réalisé, évaluation de programmes, Enseignement primaire, jeux motrices, aptitude physique, rythme corporel, vie en plein air.

\section{Perfil profesional de las autoras}

\section{Isabel Cantón Mayo}

Catedrática de Universidad de Didáctica y Organización Escolar en la Universidad de León. Sus trabajos versan sobre la calidad en la educación, la organización de centros, la gestión educativa, las Nuevas Tecnologías, la gestión del conocimiento y el Espacio Europeo de Educación Superior. Es directora del grupo GR 70 de Excelencia de la Junta de Castilla y León y Presidenta del Centro UNESCO de Castilla y León. Ha sido ponente en Jerusalén, Suecia, Buenos Aires, Francia, Perú, Costa Rica, Italia, etc.

Correo electrónico de contacto: icanm@unileon.es

\section{Argenira Carlota Ramos de Balazs}

Doctora por la Universidad de León y profesora de Educación Física en la Universidad Pedagógica Libertador de Venezuela.

Correo electrónico de contacto: argeniraramos@yahoo.com 
